\title{
Exploring the Psychometric Properties of the Disablement in the Physically Active Scale Short Form-8 in Adolescents
}

\author{
Mackenzie Holman, Madeline P. Casanova, and Russell T. Baker
}

\begin{abstract}
Context: Patient-reported outcomes are widely used in health care. The Disablement in the Physically Active (DPA) Scale Short Form-8 (SF-8) was recently proposed as a valid scale for the physically active population. However, further psychometric testing of the DPA SF-8 has not been completed, and scale structure has not been assessed using a sample of adolescent athletes. Objective: To assess scale structure of the DPA SF-8 in a sample of adolescent high-school athletes. Main Outcome Measure(s): Adolescent athletes $(\mathrm{n}=289)$ completed the DPA SF-8. Confirmatory factor analysis $(\mathrm{CFA})$ was conducted to assess the psychometric properties of the scale. Results: The CFA of the DPA SF-8 indicated that the model exceeded recommended fit indices (Comparative Fit Index $=.976$, Tucker-Lewis Index $=.965$, Root Mean Square Error of Approximation $=.061$, and Bollen's Incremental Fit Index = .976). All factor loadings were significant and ranged from .62 to .86. Modification indices did not suggest that meaningful cross-loadings were present or additional specifications that could further maximize fit or parsimony. Conclusions: The CFA of the DPA SF-8 met contemporary model fit recommendations in the adolescent athlete population. The results confirmed initial findings supporting the psychometric properties of the DPA SF-8 as well as the uniqueness of the qualityof-life and physical summary factors in an adolescent population. Further research (eg, reliability, invariance between groups, minimal clinically important differences, etc) is warranted to inform scale use in clinical practice and research.
\end{abstract}

Keywords: patient reported, outcome measures, high school

Patient-reported outcomes (PROs) allow clinicians to track and assess patient progression through the course of rehabilitation. ${ }^{1-3}$ Clinicians gain patient perceptions of health status, the impact of the injury, and the effect of interventions on different physical (eg, physical function, disability) and psychological (eg, well-being) variables throughout the course of care by using PROs. ${ }^{1-3}$ The PRO instruments may be used to capture a range of information from a generic overall health assessment to an assessment of a specific region of the body or condition. ${ }^{4}$ Thus, PROs allow clinicians to better understand the patient's view of the condition and their care. ${ }^{1,2}$ Clinicians are tasked with deciding which PRO instrument is appropriate for their patient population; clinicians should consider whether the instrument has undergone psychometric evaluation to establish its validity in the target population. Currently, there are a limited number of PROs specifically designed and validated for use in the physically active or pediatric athlete populations. ${ }^{3}$

A multidimensional instrument that is designed for the physically active population is the Disablement in the Physically Active (DPA) Scale.5,6 The DPA Scale was designed to assess multiple dimensions (eg, impairment, quality of life) of the patient's perception of his or her disablement following a musculoskeletal injury. 5,6 Although the scale has been recommended for use in practice and research, ${ }^{5-7}$ a number of concerns regarding the psychometric properties of the scale have been raised recently. ${ }^{8,9}$ Specifically, researchers noted high correlation values between hypothesized factors in the scale (eg, Impairment and Disability), suggesting multicollinearity and model misspecification. ${ }^{8,9}$ Thus, researchers recommended that modifications to the DPA Scale be made and, subsequently, proposed a short form version of the scale

The authors are with the University of Idaho, Moscow, ID, USA. Baker (russellb@ uidaho.edu) is corresponding author. (ie, the DPA SF-8). ${ }^{8}$ The psychometric issues noted in the original DPA Scale (eg, multicollinearity) were not present in the DPA SF8 , and the short version had improved model fit while also reducing patient and clinician administrative burden due to the condensed subset of items. ${ }^{8,9}$

The DPA SF- 8 retains 8 of the original 16 items in the DPA Scale and consists of two factors: a physical summary factor (PHY factor; 4 items) and a quality-of-life factor (QOL factor; 4 items). Items in the PHY factor are related to a patient's perceived physical impairment and functional limitation, whereas items in the QOL factor are related to perceived quality of life. Preliminary results have demonstrated improved psychometrics, ${ }^{8,9}$ but further testing is still needed prior to adoption into research and practice. In particular, psychometric analysis of the DPA SF-8 has not been performed using a sample of participants who only answered the 8 items; thus, the possible responses to the 8 items retained may have been influenced by the additional 8 items subsequently removed to create the DPA SF-8. Furthermore, most participants utilized in the previous study were over the age of 18 , and it is recommended to assess the scale psychometrics in an adolescent athlete population to ensure the items are appropriate for members of this demographic group. ${ }^{8-10}$ Therefore, the purpose of this study was to conduct a confirmatory factor analysis (CFA) on the DPA SF-8 to determine model fit in an adolescent athlete population.

\section{Methods}

The study was approved by the University of Idaho Institutional Review Board, and all participants provided consent prior to completing the survey; all minors provided assent and their legal guardians provided consent prior to participation. Individuals who competed in a high school-sanctioned athletic activity were recruited to complete the survey packet from three different high 
schools and a private clinic across two different states in the Pacific Northwest. The survey packet included a consent form, short demographic questionnaire, and the DPA SF-8. The demographic information collected included age, sex, sport, injury category, physical activity level, and days physically active. Demographic categories were defined using previously published definitions for injury status ${ }^{8,9}$ and physical activity level. ${ }^{11}$

Each participant received the paper survey packet and was instructed on how to complete the packet. The attending athletic trainer was permitted to explain terminology (eg, cardiovascular endurance) or answer participant questions as needed because this would naturally occur in the process of providing effective patientcentered care. The athletic trainer working with the student athletes collected the completed survey packets and then inputted the data into Qualtrics (Qualtrics, LLC, Provo, UT).

Data were downloaded from Qualtrics for analyses using Statistical Package for the Social Sciences (version 26; SPSS Inc, Chicago, IL) and SPSS Analysis of Moment Structure (version 26, AMOS; IBM Corp, Armonk, NY). Individuals who did not respond to at least $90 \%$ (ie, 7 of the 8 ) of the DPA SF-8 were removed from the data set, and any remaining missing data were replaced with the rounded mean score of the item. Data normality was assessed using histograms and skewness and kurtosis values. Univariate outliers were assessed using $z$ scores with a cutoff value of $|3.3|$ and possible multivariate outliers were identified using Mahalanobis distance at a $P<.01 .{ }^{10}$ A CFA was conducted using AMOS on the DPAS-8. Goodness-of-fit indices were evaluated using contemporary a priori values. Fit indices computed included the Comparative Fit Index ( $\geq .95)$, Tucker-Lewis Index $(\geq .95)$, Root Mean Square Error of Approximation (RMSEA) ( $\leq .06)$, and Bollen's Incremental Fit Index $(\geq .95){ }^{10}$

\section{Results}

A total of 316 individuals completed the survey packet. One individual was missing more than $10 \%$ of the DPAS and was removed from the data set; three individuals were missing one item and the rounded mean of the item was imputed for those cases. Twenty-three individuals reported scores that indicated either univariate or multivariate outliers and were removed from the dataset. A total of 289 participants (mean age $=15 \pm 1.15 \mathrm{y}$ ) were retained for analysis. Males accounted for $57.1 \%(\mathrm{n}=165)$ of the sample, whereas females accounted for $42.9 \%(n=124)$. Participants were then grouped by injury category, ${ }^{8,9}$ physical activity level,,${ }^{11}$ and days physically active (Table 1).

The CFA of the DPA met recommended model fit to the sample data (Comparative Fit Index $=.976$, Tucker-Lewis Index $=$ .965 , RMSEA $=.061$, and Bollen's Incremental Fit Index $=.976$; Figure 1). All factor loadings were significant and ranged from .62 to .86. Modification indices did not suggest there were any meaningful cross-loadings or additional specifications that could further maximize fit or parsimony.

\section{Discussion}

The purpose of our study was to examine the psychometric properties of the DPA SF-8 in the physically active adolescent athlete population. Our research is valuable for two reasons: (1) it is the first study to assess the psychometrics of the DPA SF-8 using a sample of individuals who only responded to the 8 items in this version of the scale and (2) it used a sample of individuals
Table 1 Demographic Information

\begin{tabular}{lc}
\hline Demographic variables & $\begin{array}{c}\text { Sample } \\
(\mathbf{n}=\mathbf{2 8 9})\end{array}$ \\
\hline Sex & \\
Male & $165(57.1)$ \\
Female & $124(42.9)$ \\
Age, y & $15(1.15)$ \\
Injury category & \\
Healthy & $155(53.6)$ \\
Persistent & $44(15.2)$ \\
Subacute & $38(13.1)$ \\
Acute & $41(14.2)$ \\
Unknown & $11(3.8)$ \\
Physical activity level & \\
Low & $11(3.8)$ \\
Medium & $54(18.7)$ \\
High & $189(65.4)$ \\
Unknown & $35(12.1)$ \\
Days physically active & \\
2 d & $3(1.0)$ \\
3 d & $4(1.4)$ \\
4 d & $10(3.5)$ \\
5 d & $132(45.7)$ \\
7 d & $28(9.7)$ \\
Unknown & $1(0.3)$ \\
\hline & \\
\hline &
\end{tabular}

Note: Data are presented as number of individuals (\% of total in sample). Age is given as mean (SD).

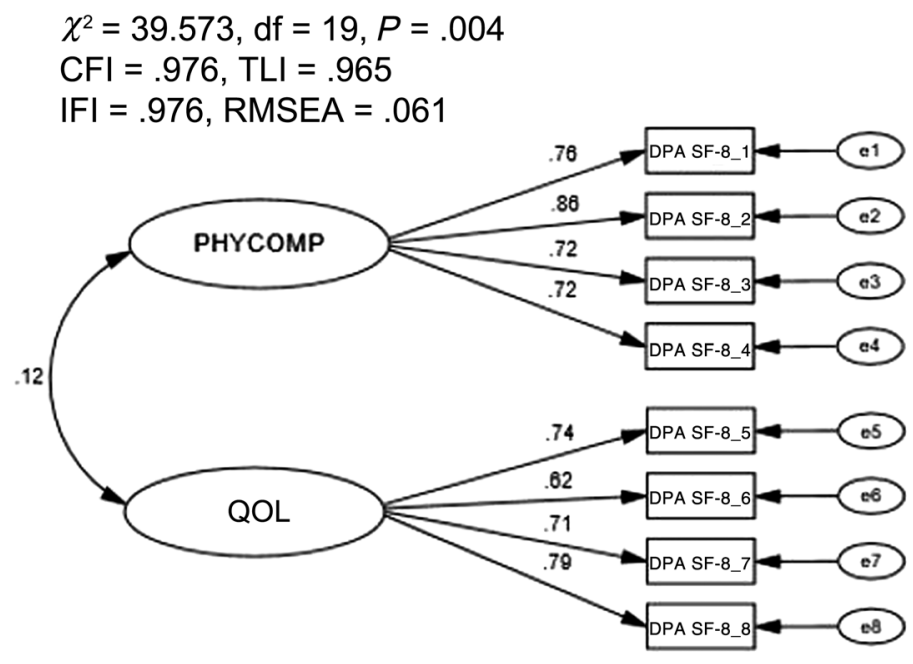

Figure $1-$ CFI of the Disablement in Physically Active Scale Short Form-8. Chi $\mathrm{Sq}=$ Chi Square $\left(\chi^{2}\right)$; CFI indicates Comparative Fit Index; IFI, Bollen's Incremental Fit Index; $P$, alpha level; QOL, quality of life; RMSEA, root mean square error of approximation; TLI, Tucker-Lewis Index.

(ie, adolescent athletes) not included in the previous DPA SF-8 research. The results of our study confirm the psychometric properties of DPA SF-8 and its appropriateness as an alternative to the DPA Scale in the adolescent athlete population. 
The DPA SF-8 exceeded the strictest contemporary model fit recommendations in an adolescent athlete population. Our results confirm previous findings ${ }^{8,9}$ that the scale does not have multicollinearity issues or model fit misspecification concerns when respondents only answer the 8 items included in the short form. Our findings were also consistent with the initial work on the DPA SF-8 supporting the QOL and PHY factors as unique dimensions that can be scored separately. ${ }^{8}$

In contrast to the previous findings, $, 6,9,9$ our findings indicated the QOL and PHY factors were not strongly correlated $(r=.12)$ in the adolescent athlete population, and the correlation was lower than what was previously reported $(r=.44-.65)$ in studies that used smaller ${ }^{6}$ and larger ${ }^{8,9}$ samples than were used in the current study. The decreased correlation between the QOL and PHY factors could be the result of sample differences (eg, size, age range, injury status, injury type, injury severity, etc) between the studies. For example, the original DPA Scale ${ }^{6}$ CFA was performed on a smaller sample that only included injured participants; our CFA was performed on a larger sample that included healthy participants. Researchers, however, have since included healthy and injured participants in DPA scale development work; healthy participants have been used to assess test-retest reliability of the DPA Scale, ${ }^{12}$ whereas healthy and injured participant samples have been used to assess other measurement properties of the DPA Scale ${ }^{7}$ and DPA SF-8. ${ }^{8,9}$ Thus, the reduced correlation between the QOL and PHY factors may be indicative of differences between the adolescent and adult populations, which supports previous research ${ }^{13}$ on QOL perception differences between the groups. In short, adolescents who suffer an injury perceive QOL disruptions and recovery differently than adults. Adults tend to weigh their QOL responses more heavily on their physical health changes, whereas mental health changes contribute more to QOL scores in adolescents. ${ }^{13}$ Overall, our results indicate that the DPA SF-8 is a parsimonious scale that is appropriate for an adolescent athlete population.

\section{Limitations and Future Research}

Although our findings support the DPA SF-8 scale structure, we must also consider the limitations of our study. Our participants were from high schools in two Pacific Northwest states, and it is possible that adolescent athletes in this region (eg, more rural students) may respond differently than adolescent athletes from different geographic areas or socioeconomic backgrounds. Thus, it may be valuable to assess participant responses from a more diverse sample of adolescent athletes. Although our CFA was performed on a larger sample than was used in previous research for the DPA Scale ${ }^{6}$ and met statistical analysis recommendations (ie, greater than 10 respondents per indicator) for the analysis, ${ }^{14}$ having a larger sample size is often recommended for instrument design research to ensure that results best represent the diverse population that may use the instrument.

Clinicians and researchers also need evidence that individuals are interpreting the DPA SF-8 items and factors similarly to allow for comparisons across time (ie, repeated measures) or group membership. The inclusion of healthy participants in the sample is valuable because clinicians use the scale to aid in determining recovery from injury (ie, informing return to play or patient discharge decisions); our results indicate that model fit of the DPA-SF 8 is sound in a sample of healthy and injured adolescent athletes. However, a limitation of our study is that the subgroup sizes do not support invariance testing between subgroups (eg, male, female; healthy, injured; etc), and we did not collect responses across repeated trials. Thus, multigroup and longitudinal invariance testing cannot be performed, which prevents confirmation that group differences or changes over time are the result of true score differences and not measurement error. ${ }^{10}$ Finally, responses collected over time would allow for other analyses (eg, reliability, minimal clinically important differences, etc) that are valuable for instrument use in research and clinical practice. Thus, future research is needed to ensure the DPA SF-8 is psychometrically sound across subgroups, reliable across time, and has a minimal clinically important difference value for use in clinical practice.

\section{Conclusions}

The CFA of the DPA SF-8 met contemporary model fit recommendations in the adolescent athlete population. Our results confirm initial findings supporting the psychometric properties of the DPA SF-8 as well as the uniqueness of the quality-of-life and physical summary factors in an adolescent population. Further research (eg, reliability, invariance between groups, minimal clinically important differences, etc) is warranted to inform scale use in clinical practice and research.

\section{References}

1. Black N. Patient reported outcome measures could help transform healthcare. BMJ. 2013;346:f167. PubMed ID: 23358487 doi:10. 1136/bmj.f167

2. Nelson EC, Eftimovska E, Lind C, Hager A, Wasson JH, Lindblad S. Patient reported outcome measures in practice. BMJ. 2015;350: g7818. PubMed ID: 25670183 doi:10.1136/bmj.g7818

3. Peroutka CR. Patient-Reported Outcomes in a Physically Active Population [dissertation]. Fargo, ND: North Dakota State University; 2018.

4. Valovich McLeod TC, Snyder AR, Parsons JT, Curtis Bay R, Michener LA, Sauers EL. Using disablement models and clinical outcomes assessment to enable evidence-based athletic training practice, part II: clinical outcomes assessment. J Athl Train. 2008; 43(4):437-445. PubMed ID: 18668177 doi:10.4085/1062-6050-43. 4.437

5. Vela LI, Denegar C. Transient disablement in the physically active with musculoskeletal injuries, part I: a descriptive model. $J$ Athl Train. 2010;45(6):615-629. PubMed ID: 21062186 doi:10.4085/ 1062-6050-45.6.615

6. Vela LI, Denegar CR. The Disablement in the Physically Active Scale, part II: the psychometric properties of an outcomes scale for musculoskeletal injuries. J Athl Train. 2010;45(6):630-641. PubMed ID: 21062187 doi:10.4085/1062-6050-45.6.630

7. Houston MN, Hoch JM, Van Lunen BL, Hoch MC. The development of summary components for the Disablement in the Physically Active scale in collegiate athletes. Qual Life Res. 2015;24(11):2657-2662. PubMed ID: 26003315 doi:10.1007/s11136-015-1007-6

8. Baker RT, Burton D, Pickering MA, Start A. Confirmatory factor analysis of the Disablement in the Physically Active Scale and preliminary testing of short-form versions: a calibration and validation study. J Athl Train. 2019;54(3):302-318. PubMed ID: 30741563 doi:10.4085/1062-6050-355-17

9. Baker RT, Casanova MP, Pickering MA, Baker JG. Invariance testing of the Disablement in the Physically Active Scale. J Athl Train. In press. doi:10.4085/1062-6050-0302.19

10. Kline RB. Principles and Practice of Structural Equation Modeling. 4th ed. New York, NY: Guilford Publications; 2015. 
11. US Department of Health and Human Services. 2008 Physical Activity Guidelines for Americans. Washington, DC: US Department of Health and Human Services; 2008.

12. Hoch JM, Druvenga B, Ferguson BA, Houston MN, Hoch MC. Patient-reported outcomes in male and female collegiate soccer players during an athletic season. J Athl Train. 2015;50(9):930-936. PubMed ID: 26207439 doi:10.4085/1062-6050-50.5.03
13. Zullig KJ, Valois RF, Drane JW. Adolescent distinctions between quality of life and self-rated health in quality of life reports. Health and Qual of Life Outcomes. 2005;3(64):1-9. doi:10.1186/1477-7525-3-64

14. Bryant FB, Yarnold PR. Principal-components analysis and exploratory and confirmatory factor analysis. In: Grimm LG, Yarnold PR (eds.) Reading and Understanding Multivariate Statistics. Washington, DC: American Psychological Association; 1995:99-136. 